\title{
Alternative Secondary Goals in Multiplicative Two-Stage Data Envelopment Analysis
}

\author{
Chao Lu (i) and Haifang Cheng \\ School of Management, Huazhong University of Science and Technology, Wuhan 430074, China \\ Correspondence should be addressed to Haifang Cheng; chenghf@hust.edu.cn
}

Received 29 March 2021; Accepted 29 May 2021; Published 9 June 2021

Academic Editor: Josefa Mula

Copyright ( $) 2021$ Chao Lu and Haifang Cheng. This is an open access article distributed under the Creative Commons Attribution License, which permits unrestricted use, distribution, and reproduction in any medium, provided the original work is properly cited.

\begin{abstract}
Data envelopment analysis (DEA) is a nonparametric method for evaluating the relative efficiency of a set of decision-making units (DMUs) with multiple inputs and outputs. As an extension of the DEA, a multiplicative two-stage DEA model has been widely used to measure the efficiencies of two-stage systems, where the first stage uses inputs to produce the outputs, and the second stage then uses the first-stage outputs as inputs to generate its own outputs. The main deficiency of the multiplicative twostage DEA model is that the decomposition of the overall efficiency may not be unique because of the presence of alternate optima. To remove the problem of the flexible decomposition, in this paper, we maximize the sum of the two-stage efficiencies and simultaneously maximize the two-stage efficiencies as secondary goals in the multiplicative two-stage DEA model to select the decomposition of the overall efficiency from the flexible decompositions, respectively. The proposed models are applied to evaluate the performance of 10 branches of China Construction Bank, and the results are compared with the results of the existing models.
\end{abstract}

\section{Introduction}

Data envelopment analysis (DEA) that was introduced by Charnes et al. is a nonparametric method for evaluating the relative efficiency of a set of decision-making units (DMUs) with multiple inputs and outputs [1]. One of the important areas of development for DEA models has been devoted to applications wherein the DMUs have two-stage processes [2]. In the first stage, inputs are used to produce outputs, and the second stage then uses the first-stage outputs, which are referred to as intermediate measures, as inputs to generate its own outputs. For example, Seiford and Zhu examined the profitability and marketability of the top 55 US commercial banks via a two-stage process [3]. In the first stage for profitability, labor and assets are used as inputs, and the outputs are profits and revenue. In the second stage for marketability, the profits and revenue are then used as inputs to generate its outputs: market value, returns, and earnings per share. Similar two-stage processes have also been studied in other applications, e.g., nonlife insurance companies by
Kao and Hwang [4], nonstorable transport services by Chiou et al. [5], sustainable product design by Chen et al. [6], NBA teams by Yang et al. [7], hotels by Huang et al. [8] and Yin et al. [9], banking system by Wang et al. [10], Wanke and Barros [11], and Henriques et al. [12], energy performance by Wang et al. [13] and Zhu et al. [14], supply chain management by Tajbakhsh et al. [15] and Tavana et al. [16], fixed cost allocation by Yu et al. [17], and the sustainability of industrial sectors by Zhu et al. [18]. To see the main twostage model and applications, we refer the readers to the review papers of Kao and Halkos et al. [19, 20].

Halkos et al. classified the existing two-stage DEA models into four categories: independent two-stage DEA, connected two-stage DEA, relational two-stage DEA, and game theoretic two-stage DEA models [20]. Recently, a few researchers have focused mainly on the study of the relational two-stage DEA model, where a mathematical relationship between the overall efficiency and the two-stage efficiencies is either multiplicative or additive, and the intermediate measure uses the same set of weights in the two 
stages. The models that appeared in the literature are the multiplicative model by Kao and Hwang [4], and the additive models by Chen et al. [21], Wang and Chin [22], and Chiou et al. [5].

In the additive model, Chen et al. and Guo et al. modeled the overall efficiency as the weighted sum of the two individual efficiencies [21, 23]. If appropriate values for the weights of two stages are chosen, the model can then also be converted into a linear form; otherwise, it cannot be turned into a linear program. If the weights of two stages are equal, Chen et al.'s model then becomes Chiou et al.'s model, which is proved to be a nonconvex optimization problem by Lim and $\mathrm{Zhu}$ [24]. Lim and Zhu have developed a procedure to obtain an approximate global optimal solution to Chiou et al.'s model based on Liang et al.'s method with an improved search bound $[5,25]$. Halkos et al. modified the original model of Chen et al. by incorporating assurance region-like constraints to the weights of the model $[21,26]$. This helps the decision maker to choose the available region for the values of the weights. The new weight assurance region model guarantees that the model can always be turned into a linear one.

In the multiplicative model, Kao and Hwang defined the overall efficiency of all of the DMUs as the product of the two individual efficiencies [4]. By applying Charnes and Cooper's transformation, the multiplicative model can be converted into a linear program. Kao and Hwang's model is limited by the assumption of constant returns to scale, Wang and Chin demonstrate that the multiplicative two-stage DEA model of Kao and Hwang can be easily extended to variable returns to scale assumption [22]. Kao and Hwang further decompose the system efficiency into the overall technical and scale efficiencies, where the overall technical and scale efficiencies are the products of the corresponding efficiencies of the two processes, respectively [27]. Chen et al. prove that Chen and Zhu's model transformed in constant returns to scale is equivalent with Kao and Hwang's model [4, 21, 28]. The advantage of Kao and Hwang's model is the assessment of individual efficiencies for the two stages. However, as Kao and Hwang have mentioned, the optimal solutions from the multiplicative model of a linear form may not be unique [4]. Consequently, neither will be the decomposition of the overall efficiency. Thus, how to select the decomposition of the overall efficiency from the flexible decompositions is essential for the decision maker.

To obtain the decomposition of the overall efficiency, Kao et al. and Liang et al. both suggested obtaining an optimal solution, which produces the largest individual efficiency score in first- or second-stage while maintaining the overall efficiency score that is unchanged $[4,29]$. Because the calculation of Kao et al.'s model requires two models solved in two stages, Liu developed a big-M method to combine these into one and find the two individual efficiencies and overall efficiencies simultaneously [30]. Based on Kao et al.'s model, Zhou et al. view the two stages as two parties and develop a Nash bargaining game model to obtain a fair efficiency decomposition for the two stages while keeping the overall efficiency unchanged [31]. However, Kao et al.'s approach of maximizing the efficiency of one stage means the stage is indeed the leader and the other stage is just a follower, and this can run counter to the original intention [31]. If the powers of both stages are balanced, it is reasonable for them to select the decomposition of the overall efficiency from the flexible decompositions by maximizing simultaneously the two individual efficiencies. This decomposition method considers the fairness of total efficiency decomposition, which is extremely necessary because if any stage's divisional efficiency is obtained with unfairness, conflicts between the two stages may occur. Furthermore, Zhou et al.'s model is available in the case in which the two stages belong to two different organizations (e.g., manufacturer, retailer). If two stages belong to one organization (e.g., commercial bank), or different organizations that need cooperation, then the decision makers may hope that the efficiencies sum of the two stages achieve the maximum. The objective function of this decomposition method is consistent with that of the additive two-stage DEA model. In this paper, alternative secondary goals, which are maximizing the sum of the two individual efficiencies and maximizing simultaneously the two individual efficiencies, are introduced into the multiplicative two-stage DEA model, respectively, to select the decomposition of the overall efficiency from the flexible decompositions.

The rest of the paper is organized as follows: Section 2 briefly reviews the multiplicative two-stage DEA models as well as the existing secondary goals in DEA models. Two proposed models and their solution methods are introduced in Section 3. Section 4 illustrates the proposed models using a numerical example and then compares the results with the results of the existing models. Conclusions are provided in Section 5.

\section{Background and Prior Research}

2.1. Multiplicative Two-Stage DEA Model. There are two types of relational two-stage DEA models, including the multiplicative two-stage DEA model proposed by Kao and Hwang and the additive two-stage DEA model proposed by Chiou et al. $[4,5]$. In this section, we briefly review the multiplicative twostage DEA model related with the proposed models.

Consider a two-stage process with IDMUs. Assume that each $\mathrm{DMU}_{k}(k=1,2, \ldots, I)$ uses inputs $x_{k}=$ $\left(x_{k 1}, x_{k 2}, \ldots, x_{k J}\right)^{T}$ to produce the outputs $z_{k}=$ $\left(z_{k 1}, z_{k 2}, \ldots, z_{k R}\right)^{T}$ in the first stage. Then, these outputs become the inputs of the second stage and are referred to as intermediate measures. The outputs from the second stage are denoted as $y_{k}=\left(y_{k 1}, y_{k 2}, \ldots, y_{k S}\right)^{T}$. The two individual efficiencies $E_{k}^{1}$ and $E_{k}^{2}$ for the $\mathrm{DMU}_{k}$ in the first and second stages can be measured independently using equations (1) and (2), respectively:

$$
\begin{aligned}
& E_{k}^{1}=\frac{w^{T} z_{k}}{v^{T} x_{k}}, \\
& E_{k}^{2}=\frac{u^{T} y_{k}}{w^{T} z_{k}},
\end{aligned}
$$


where $v=\left(v_{1}, v_{2}, \ldots, v_{I}\right)^{T}$ and $w=\left(w_{1}, w_{2}, \ldots, w_{R}\right)^{T}$ are the input and output weights in the first stage. Because the input of the second stage will be the expected output of the first stage, it is assumed that $w$ is also the input weight in the second stage. The output weight in the second stage is denoted by $u=\left(u_{1}, u_{2}, \ldots, u_{S}\right)^{T}$. To calculate the overall efficiency, some models must be used to describe the relationships between the whole process and the two subprocesses. In the existing literature, these models are the multiplicative model and the additive model. As Chen et al. explained, the multiplicative and additive models are two different but equally valid ways of aggregating the components of a two-stage process [21].

In the multiplicative two-stage DEA model, Kao and Hwang define the overall efficiency of the $\mathrm{DMU}_{k}$ as the product of the two individual efficiencies, and their model for measuring the overall efficiency of the $\mathrm{DMU}_{k}$ is given as follows [4]:

$$
\begin{aligned}
& \max E_{k p}=\frac{w^{T} z_{k}}{v^{T} x_{k}} \times \frac{u^{T} y_{k}}{w^{T} z_{k}}=\frac{u^{T} y_{k}}{v^{T} x_{k}} \\
& \text { s.t. } \frac{w^{T} z_{i}}{v^{T} x_{i}} \leq 1, \quad i=1,2, \ldots, I \\
& \frac{u^{T} y_{i}}{w^{T} z_{i}} \leq 1, \quad i=1,2, \ldots, I \\
& v \geq 0, u \geq 0, w \geq 0,
\end{aligned}
$$

where $E_{k p}$ represents the multiplicative efficiency for measuring the overall efficiency of the $\mathrm{DMU}_{k}$. By applying Charnes and Cooper's transformation, model (3) can be converted into the following linear program:

$$
\begin{array}{ll}
\max & E_{k p}=u^{T} y_{k} \\
\text { s.t. } & v^{T} x_{k}=1 \\
& w^{T} z_{i}-v^{T} x_{i} \leq 0, \quad i=1,2, \ldots, I \\
& u^{T} y_{i}-w^{T} z_{i} \leq 0, \quad i=1,2, \ldots, I \\
& v \geq 0, u \geq 0, w \geq 0 .
\end{array}
$$

After the optimal solutions $v^{*}, u^{*}$, and $w^{*}$ are solved, the multiplicative efficiency score of the $\mathrm{DMU}_{k}$ is computed subsequently as $E_{k p}^{*}=u^{* T} y_{k}$. Using equations (1) and (2), the two individual efficiency scores $E_{k}^{1 *}$ and $E_{k}^{2 *}$ can be obtained.

However, the optimal solutions of model (4) may not be unique. Consequently, the decomposition of the overall efficiency will not be unique [4]. As a result, the comparison of either $E_{k}^{1}$ or $E_{k}^{2}$ among all of the DMUs lacks a common basis. One solution proposed by Kao and Hwang for this problem is to find the optimal solution, which produces the largest individual efficiency in the first stage or second stage while maintaining the overall efficiency value at $E_{k p}^{*}$, which is calculated from model (4). This idea can be formulated as

$$
\begin{array}{ll}
\max & E_{k}^{1}=w^{T} z_{k} \\
\text { s.t. } & E_{k p}^{*}=u^{T} y_{k} \\
& v^{T} x_{k}=1 \\
& w^{T} z_{i}-v^{T} x_{i} \leq 0, \quad i=1,2, \ldots, I \\
& u^{T} y_{i}-w^{T} z_{i} \leq 0, \quad i=1,2, \ldots, I \\
& v \geq 0, u \geq 0, w \geq 0,
\end{array}
$$

or

$$
\begin{array}{ll}
\max & E_{k}^{2}=u^{T} y_{k} \\
\text { s.t. } & w^{T} z_{k}=1 \\
& u^{T} y_{k}-E_{k p}^{*} v^{T} x_{k}=0 \\
& w^{T} z_{i}-v^{T} x_{i} \leq 0, \quad i=1,2, \ldots, I \\
& u^{T} y_{i}-w^{T} z_{i} \leq 0, \quad i=1,2, \ldots, I \\
& v \geq 0, u \geq 0, w \geq 0 .
\end{array}
$$

Once the individual maximal achievable efficiency score $\bar{E}_{k}^{1}$ or $\bar{E}_{k}^{2}$ is obtained using models (5) or (6), the other minimal achievable efficiency score can be computed using $\underline{E}_{k}^{2}=E_{k p}^{*} / \bar{E}_{k}^{1}$ or $\underline{E}_{k}^{1}=E_{k p}^{*} / \bar{E}_{k}^{2}$. If $\bar{E}_{k}^{1} \neq \underline{E}_{k}^{1}$ or $\bar{E}_{k}^{2} \neq \underline{E}_{k}^{2}$, the efficiency decomposition is not unique, implying that some flexibility may exist in decomposing the overall efficiency between the two stages.

2.2. The Existing Secondary Goals in DEA Models. The main problem of the DEA model is the nonuniqueness of the optimal solutions; therefore, it is recommended that secondary goals be introduced in the DEA model to be used for choosing weights from the optimal solutions of the model. In the one-stage DEA model, various forms of secondary goals are proposed to deal with the nonuniqueness issue, for example, selecting symmetric weights as a secondary goal, optimizing the rank position of the DMU as secondary goal [32-34]. Soltanifar and Shahghobadi first review the existing secondary goals and then extend the idea of some authors to construct three new secondary goals. They also propose a new approach to select the best secondary goal among the mentioned secondary goals by using a voting model [35].

As mentioned before, in the multiplicative two-stage DEA model (4), the optimal solutions may not be unique. Consequently, the decomposition of the overall efficiency will not be unique. To obtain the decomposition of the overall efficiency from the flexible decompositions, Kao and Hwang propose models (5) and (6), in which maximizing individual efficiency in the first stage or second stage is used as a secondary goal in the multiplicative two-stage DEA model, respectively [4]. Zhou et al., viewing the two stages as two parties and using the minimal achievable efficiencies of the two stages as the breakdown points, develop a Nash bargaining game model to search for the decomposition of the overall efficiency that is as fair as possible for both parties, and the result shows that the bargaining results are a 
tradeoff between the minimal and maximal achievable efficiencies for each stage [31]. Tavana et al. [36] use a bargaining game approach to decompose efficiency in two-stage DEA models with uncertain inputs, intermediate measures, and outputs. An et al. [37] use a multiple-objective programming model to obtain the maximum efficiencies of a system and its stages simultaneously. In their model, the ratio of two stages' individual efficiencies of a DMU are set as the reference point for the DMU's efficiency decomposition, and the goal is to make the ratio of decomposed divisional efficiencies close to the reference point. Fang [38] develop a methodology for assessing the overall and stage efficiencies by considering the different and DMU-specific degree of priority given to the stages. In Table 1 , we provide an overview of existing secondary goals related literature in DEA.

The choice of secondary goals depends on two aspects: the relationship between two stages and decision maker's preference in the particular application. If one stage is the leader and the other one is a follower, then Kao and Hwang's decomposition method should be used; if we view the two stages as two parties, then the Nash bargaining game model proposed by Zhou et al. should be used [4]. If two stages belong to one organization, then the decision makers may hope that the efficiencies of the two stages all achieve the maximum. In the following, two new secondary goals, $\max \left(E_{k}^{1}+E_{k}^{2}\right)$ and $\max \left\{E_{k}^{1}, E_{k}^{2}\right\}$, are proposed in the multiplicative two-stage DEA model to select the decomposition of the overall efficiency from the flexible decompositions, respectively.

\section{The Proposed Secondary Goals in Multiplicative Two-Stage DEA Models}

3.1. Maximizing the Sum of Two Individual Efficiencies as a Secondary Goal. We first maximize the sum of two individual efficiencies as a secondary goal in the multiplicative two-stage DEA model. Given the optimal multiplicative efficiency $E_{k p}^{*}$ determined by model (4), this model can be formulated as

$$
\begin{aligned}
& \max E_{k a}=\frac{w^{T} z_{k}}{v^{T} x_{k}}+\frac{u^{T} y_{k}}{w^{T} z_{k}} \\
& \text { s.t. } u^{T} y_{k}-E_{k p}^{*} v^{T} x_{k}=0 \\
& w^{T} z_{i}-v^{T} x_{i} \leq 0, \quad i=1,2, \ldots, I \\
& u^{T} y_{i}-w^{T} z_{i} \leq 0, \quad i=1,2, \ldots, I \\
& v \geq 0, u \geq 0, w \geq 0 .
\end{aligned}
$$

Let $v^{T} x_{k}=1$; then, model (7) can be rewritten as

$$
\begin{aligned}
& \max E_{k a}=w^{T} z_{k}+\frac{E_{k p}^{*}}{w^{T} z_{k}} \\
& \text { s.t. } v^{T} x_{k}=1 \\
& u^{T} y_{k}-E_{k p}^{*}=0 \\
& w^{T} z_{i}-v^{T} x_{i} \leq 0, \quad i=1,2, \ldots, I \\
& u^{T} y_{i}-w^{T} z_{i} \leq 0, \quad i=1,2, \ldots, I \\
& v \geq 0, u \geq 0, w \geq 0 .
\end{aligned}
$$
becomes

Let $w^{T} z_{k}=E_{k}^{1}$; then, similar to Zhou et al. [31], model (8)

$$
\begin{array}{ll}
\max & E_{k a}=E_{k}^{1}+\frac{E_{k p}^{*}}{E_{k}^{1}} \\
\text { s.t. } \quad E_{k}^{1} \leq E_{k}^{1} \leq \bar{E}_{k}^{1} .
\end{array}
$$

The maximal and minimal achievable efficiency scores $\bar{E}_{k}^{1}$ and $\underline{E}_{k}^{1}$ for the first stage can be obtained by solving models (5) and (6), respectively.

It is obvious that the objective function in model (9) is convex, so the maximum value of model (9) is reached at the two sides of the interval $\left[\underline{E}_{k}^{1}, \bar{E}_{k}^{1}\right]$. Thus, we can first find the maximal and minimal achievable efficiency scores of the first stage by solving models (5) and (6), respectively. We then can obtain the optimal solution of model (9) by comparing the corresponding objective function values. This procedure is described as follows:

Step 1: find the maximal achievable efficiency score $\bar{E}_{k}^{1}$ of the first stage.

Solve model (5) and obtain the optimal solutions $u^{(1)}$, $v^{(1)}$, and $w^{(1)}$. Then, the maximal achievable efficiency score of the first stage is calculated as $\bar{E}_{k}^{1}=w^{(1) T} z_{k}$, and the minimal achievable efficiency score of the second stage is calculated as $\underline{E}_{k}^{2}=E_{k p}^{*} / \bar{E}_{k}^{1}$.

Step 2: find the minimal achievable efficiency score $\underline{E}_{k}^{1}$ of the first stage.

Solve model (6) and obtain the optimal solutions $u^{(2)}$, $v^{(2)}$, and $w^{(2)}$. Then, the maximal achievable efficiency score of the second stage is calculated as $\bar{E}_{k}^{2}=u^{(2) T} y_{k}$, and the minimal achievable efficiency score of the first stage is calculated as $\underline{E}_{k}^{1}=E_{k p}^{*} / \bar{E}_{k}^{2}$.

Step 3: compare the objective function values to obtain the optimal solution.

To obtain the optimal solution of model (9), we first apply the following equation to determine the optimal objective function value $E_{k a}^{*}$ : 
TABLE 1: The existing secondary goals in DEA models.

\begin{tabular}{ll}
\hline References & Secondary goal
\end{tabular}

One-stage DEA model

Dimitrov and Sutton [32] and

Jahanshahloo et al. [33]

Contreras [34]

Liang et al. [39] and Wang and Chin [40]

$\mathrm{Wu}$ et al. [41]

Two-stage DEA model

Kao and Hwang [4] and Liang et al. [29]

Zhou et al. [31] and Tavana et al. [36]

An et al. [37]

Fang [38]
Promoting symmetric weight selection

Optimizing the rank position of the DMU

Minimizing the total deviation from the ideal point, minimizing the maximum d-efficiency score, minimizing the mean absolute deviation Selecting weights based on both desirable and undesirable cross-efficiency targets

Maximizing one stage's efficiency

Minimizing the individual efficiency of the other party

Making the ratio of decomposed divisional efficiencies close to the reference point Assessing the stage efficiencies by considering the different and DMU-specific degree of priority given to the stages.

$$
E_{k a}^{*}=\max \left\{\bar{E}_{k}^{1}+\underline{E}_{k}^{2}, \underline{E}_{k}^{1}+\bar{E}_{k}^{2}\right\}=\max \left\{\bar{E}_{k}^{1}+\frac{E_{k p}^{*}}{\bar{E}_{k}^{1}}, \underline{E}_{k}^{1}+\frac{E_{k p}^{*}}{\underline{E}_{k}^{1}}\right\} .
$$

If $\bar{E}_{k}^{1}+\left(E_{k p}^{*} / \bar{E}_{k}^{1}\right) \neq \underline{E}_{k}^{1}+\left(E_{k p}^{*} / \underline{E}_{k}^{1}\right)$, or $\bar{E}_{k}^{1} \underline{E}_{k}^{1} \neq E_{k p}^{*}$, we then can identify that the optimal solutions $u^{*}, v^{*}$, and $w^{*}$ as well as $E_{k}^{1 *}$ and $E_{k}^{2 *}$ of model (9) come from either step 1 or 2, which shows that an efficiency decomposition can obtained by solving the proposed model (7). Otherwise, there are two efficiency decompositions: one is come from step 1 and the other from step 2. At this time, we can obtain the efficiency decomposition by considering the relative importance of the performances of the two stages to the overall performance of the DMUs.

If the relative importance or the contribution of the performances is considered, the secondary goal in model (7) can then be modified as follows:

$$
\max E_{k a}=\xi_{1} \cdot \frac{w^{T} z_{k}}{v^{T} x_{k}}+\xi_{2} \cdot \frac{u^{T} y_{k}}{w^{T} z_{k}},
$$

where $\xi_{1}$ and $\xi_{2}$ are the user-specified weights such that $0 \leq \xi_{1}, \xi_{2} \leq 1$, and $\xi_{1}+\xi_{2}=1$. Similar to the above analysis, we can obtain the optimal solution of the model, where the secondary goal is to maximize the weighted sum for the efficiencies of the individual stages. We first use the following equation to determine the optimal objective function value $E_{k a}^{*}$ :

$$
\begin{aligned}
E_{k a}^{*} & =\max \left\{\xi_{1} \bar{E}_{k}^{1}+\xi_{2} \underline{E}_{k}^{2}, \xi_{1} \underline{E}_{k}^{1}+\xi_{2} \bar{E}_{k}^{2}\right\} \\
& =\max \left\{\xi_{1} \bar{E}_{k}^{1}+\xi_{2} \frac{E_{k p}^{*}}{\bar{E}_{k}^{1}}, \xi_{1} \underline{E}_{k}^{1}+\xi_{2} \frac{E_{k p}^{*}}{\underline{E}_{k}^{1}}\right\} .
\end{aligned}
$$

We then can determine the optimal solutions $u^{*}, v^{*}$, and $w^{*}$, as well as $E_{k}^{1 *}$ and $E_{k}^{2 *}$, using the same approach that was described in Step 3 .

3.2. Maximizing Simultaneously the Two Individual Efficiencies as a Secondary Goal. Similar to the analysis in
Section 3.1, if the secondary goal is to simultaneously maximize the two individual efficiencies after the optimal multiplicative efficiency $E_{k p}^{*}$ was determined by model (4), then the optimization model for determining the efficiency decomposition can be expressed as the following bi-objective programming model:

$$
\begin{aligned}
& \max E_{k m}=\left\{E_{k}^{1}, \frac{E_{k p}^{*}}{E_{k}^{1}}\right\} \\
& \text { s.t. } \quad E_{k}^{1} \leq E_{k}^{1} \leq \bar{E}_{k}^{1} .
\end{aligned}
$$

Model (13) is a nonlinear model, which is equivalent to the following linear form:

$$
\begin{aligned}
& \max E_{k m 1}=E_{k}^{1}, \\
& \min E_{k m 2}=E_{k}^{1}, \\
& \text { s.t. } \underline{E}_{k}^{1} \leq E_{k}^{1} \leq \bar{E}_{k}^{1} .
\end{aligned}
$$

We use the fuzzy programming method to solve model (14) even though other methods can also be used to solve it. In model (14), it is unlikely that the two objectives will simultaneously achieve their optimum when subjected to the given constraints. So, in practice, some satisfying solutions are usually chosen according to the aspiration level that is fixed for each objective [42].

In model (14), $\bar{E}_{k}^{1}$ and $\underline{E}_{k}^{1}$ can be specified as the aspiration level for the two objectives, respectively. Thus, model (14) can be expressed as follows:

$$
\begin{aligned}
& \text { Find } E_{k}^{1} \text {, } \\
& \text { To satisfy } E_{k}^{1} \widetilde{\widetilde{E}} \bar{E}_{k}^{1}, E_{k}^{1} \widetilde{\widetilde{E}} \underline{E}_{k}^{1}, \underline{E}_{k}^{1} \leq E_{k}^{1} \leq \bar{E}_{k}^{1} .
\end{aligned}
$$

The expressions $E_{k}^{1} \geq \bar{E}_{k}^{1}$ and $E_{k}^{1} \leq \underline{E}_{k}^{1}$ are represented by a fuzzy set called the fuzzy goal, which means that the decision maker would be satisfied even if the first objective value is slightly less than $\bar{E}_{k}^{1}$ and the second objective value is slightly greater than $\underline{E}_{k}^{1}$ within the value of allowed deviations. The symbols " $\geq, \leq$ " denote the fuzzified versions of " $\geq$, $\leq$ ", and they can be read as "approximately greater/less than or equal to". 
The procedure for finding the fuzzy efficient solution of model (15) can be summarized as follows:

Step 1: construct the membership functions.

In model (15), two fuzzy goals are represented by fuzzy sets and are defined by membership functions $\mu_{1}\left(E_{k}^{1}\right)$ and $\mu_{2}\left(E_{k}^{1}\right)$, respectively. Because linear membership functions are used in the literature and are practiced more than the other types of membership functions, we choose the linear membership functions [43]. Hence, the membership functions for the maximization and minimization objectives may be defined, respectively, as follows:

$$
\begin{aligned}
& \mu_{1}\left(E_{k}^{1}\right)=\left\{\begin{array}{l}
0, \quad E_{k}^{1} \leq \underline{E}_{k}^{1}, \\
\frac{E_{k}^{1}-\underline{E}_{k}^{1}}{\bar{E}_{k}^{1}-\underline{E}_{k}^{1}}, \underline{E}_{k}^{1} \leq E_{k}^{1} \leq \bar{E}_{k}^{1}, 1, E_{k}^{1} \geq \bar{E}_{k}^{1},
\end{array}\right. \\
& \mu_{2}\left(E_{k}^{1}\right)=\left\{\begin{array}{l}
1, \\
\bar{E}_{k}^{1}-E_{k}^{1} \\
\frac{\bar{E}_{k}^{1}-\underline{E}_{k}^{1}}{\bar{E}_{k}^{1} \leq E_{k}^{1},}, \bar{E}_{k}^{1}, 0, E_{k}^{1} \geq \bar{E}_{k}^{1} .
\end{array}\right.
\end{aligned}
$$

Step 2: build an auxiliary crisp model.

Introducing an auxiliary variable $\lambda$ and using the max-min's approach, model (15) is transformed into the following auxiliary crisp model:

$\operatorname{Max} \lambda$

$$
\begin{array}{ll}
\text { s.t. } & \frac{E_{k}^{1}-\underline{E}_{k}^{1}}{\bar{E}_{k}^{1}-\underline{E}_{k}^{1}} \geq \lambda \\
& \frac{\bar{E}_{k}^{1}-E_{k}^{1}}{\bar{E}_{k}^{1}-\underline{E}_{k}^{1}} \\
& \underline{E}_{k}^{1} \leq E_{k}^{1} \leq \bar{E}_{k}^{1}, \quad 0 \leq \lambda \leq 1 .
\end{array}
$$

The optimal solution to model (18) is a fuzzy efficient solution to model (15) and the Pareto optimal solution of model (14).

Step 3: solve model (18) to find the optimal solution.

Model (18) is a linear programming model with two variables $\lambda$ and $E_{k}^{1}$. Using the graphic method to solve it, we obtain the optimal solution $\lambda^{*}=(1 / 2)$ and $E_{k}^{1 *}=\left(\underline{E}_{k}^{1}+\bar{E}_{k}^{1} / 2\right)$. Then, the optimal efficiency score of the second stage is calculated as $E_{k}^{2 *}=\left(E_{k p}^{*} / E_{k}^{1 *}\right)=\left(2 E_{k p}^{*} / E_{k}^{1}+\bar{E}_{k}^{1}\right)$. It means that maximizing simultaneously the two-stage efficiencies as secondary goals in the multiplicative two-stage DEA model can also obtain the efficiency decomposition from the flexible decompositions.

The above max-min's approach for transforming model (15) into model (18) does not consider the relative importance of the two objective functions. If the objectives are not equally important, using a weighted $\max -\min$ approach, model (18) can then be modified as

$$
\begin{array}{ll}
\operatorname{Max} \lambda & \\
\text { s.t. } & \frac{E_{k}^{1}-\underline{E}_{k}^{1}}{\bar{E}_{k}^{1}-\underline{E}_{k}^{1}} \geq \gamma_{1} \lambda \\
& \frac{\bar{E}_{k}^{1}-E_{k}^{1}}{\bar{E}_{k}^{1}-\underline{E}_{k}^{1}} \geq \gamma_{2} \lambda \\
& \underline{E}_{k}^{1} \leq E_{k}^{1} \leq \bar{E}_{k}^{1}, \quad 0 \leq \lambda \leq 1,
\end{array}
$$

where $\gamma_{1}$ and $\gamma_{2}$ are the weights that present the relative importance of the two fuzzy goals, $0 \leq \gamma_{1}, \gamma_{2} \leq 1$, and $\gamma_{1}+\gamma_{2}=1$. This model finds an optimal solution within the feasible area such that the ratio of the achievement levels is as close to the ratio of the objective weights as possible [44].

Model (19) is also a linear programming model with two variables $\lambda$ and $E_{k}^{1}$. Using the graphic method to solve it, we obtain the optimal solution $\lambda^{*}=1$ and $E_{k}^{1 *}=\gamma_{1} \bar{E}_{k}^{1}+\gamma_{2} E_{k}^{1}$. Then, the optimal efficiency score of the second stage is calculated as $E_{k}^{2 *}=\left(E_{k p}^{*} / E_{k}^{1 *}\right)=\left(E_{k p}^{*} / \gamma_{1} \bar{E}_{k}^{1}+\gamma_{2} E_{k}^{1}\right)$.

\section{A Numerical Example}

In this section, a numerical example is used to illustrate the proposed method. In order to compare the results determined by the proposed model with the results of the existing model, a numerical example used by Yang et al. [45] and Zhou [31] et al. is presented. There are 10 bank branches of China Construction Bank (CCB) in the Anhui province, PR China, and their process can be modeled as a two-stage system. In the first stage, an employee (EM), fixed assets (FAs), and expenses (EXs) are consumed to generate outputs: credit (CR) and interbank loans (ILs). In the second stage, the outputs from the first stage are used as inputs to generate outputs: loan (LO) and profit (PR). The dataset for the 10 bank branches is listed in Table 2 .

In this example, Zhou et al. have calculated the overall efficiency scores and the maximal and minimal achievable efficiency scores of the two stages for each DMU using models (4)-(6), and the results are given in Table 3 [31]. All of the corresponding rank values for each DMU are listed in parentheses. The thirteenth to the sixteenth rows of Table 3 detail the descriptive statistics for the efficiency scores of 10 bank branches determined by Kao and Hwang's models [4]. It shows that only the $\mathrm{DMU}_{1}$ performed efficiently in both stages and in the whole system. For the remaining nine DMUs, because the maximal and minimal achievable efficiency scores for each stage are not the same, some flexibility exists in the efficiency decomposition between the two stages for those DMUs. Therefore, it is necessary to use a secondary goal to select the reasonable decomposition of the overall efficiency from the flexible decompositions.

We first apply model (9) to determine the solution of the proposed model (7). The results, including two individual 
TABle 2: Dataset of 10 bank branches (reproduced from Zhou et al., 2013).

\begin{tabular}{|c|c|c|c|c|c|c|c|c|}
\hline \multicolumn{2}{|c|}{ DMU } & \multicolumn{3}{|c|}{ Inputs $(X)$} & \multicolumn{2}{|c|}{ Intermediate $(Z)$} & \multicolumn{2}{|c|}{ Outputs $(Y)$} \\
\hline No. & Branch & $\operatorname{EM}\left(10^{3}\right)$ & FA $\left(¥ 10^{8}\right)$ & $\mathrm{EX}\left(¥ 10^{8}\right)$ & $\mathrm{CR}\left(¥ 10^{8}\right)$ & IL $\left(¥ 10^{8}\right)$ & LO $\left(¥ 10^{8}\right)$ & $\mathrm{PR}\left(¥ 10^{8}\right)$ \\
\hline 1 & Maanshan & 0.526 & 0.4775 & 0.3848 & 49.9174 & 5.4613 & 34.9897 & 0.8430 \\
\hline 2 & Anqing & 0.713 & 1.2363 & 0.5547 & 37.4954 & 4.0825 & 20.6013 & 0.4864 \\
\hline 3 & Huangshan & 0.443 & 0.4460 & 0.3419 & 20.9846 & 0.6897 & 8.6332 & 0.1288 \\
\hline 4 & Fuyang & 0.638 & 1.2481 & 0.4574 & 45.0508 & 1.7237 & 9.2354 & 0.3019 \\
\hline 5 & Suzhou & 0.575 & 0.7050 & 0.4036 & 38.1625 & 2.2492 & 12.0171 & 0.3138 \\
\hline 6 & Chuzhou & 0.432 & 0.6446 & 0.4012 & 30.1676 & 2.3354 & 13.813 & 0.3772 \\
\hline 7 & Luan & 0.510 & 0.7239 & 0.3709 & 26.5391 & 1.3416 & 5.0961 & 0.1453 \\
\hline 8 & Chizhou & 0.322 & 0.3363 & 0.2334 & 16.1235 & 0.4889 & 5.9803 & 0.0928 \\
\hline 9 & Chaohu & 0.423 & 0.6678 & 0.3471 & 22.1848 & 1.1767 & 9.2348 & 0.2002 \\
\hline 10 & Bozhou & 0.256 & 0.3418 & 0.1594 & 13.4364 & 0.4064 & 2.5326 & 0.0057 \\
\hline
\end{tabular}

TABle 3: Efficiency scores and ranks of 10 bank branches determined by Kao and Hwang's models.

\begin{tabular}{lccccc}
\hline \multirow{2}{*}{ DMU } & Model (4) & \multicolumn{2}{c}{ Model (5) } & \multicolumn{2}{c}{ Model (6) } \\
& $E_{k p}^{*}$ & $\bar{E}_{k}^{1}$ & $\underline{E}_{k}^{2}$ & $\underline{E}_{k}^{1}$ & $\bar{E}_{k}^{2}$ \\
\hline 1 & $1.0000(1)$ & $1.0000(1)$ & $1.0000(1)$ & $1.0000(1)$ & $1.0000(1)$ \\
2 & $0.4344(3)$ & $0.5541(6)$ & $0.7838(2)$ & $0.5526(2)$ & $0.7861(8)$ \\
3 & $0.2930(7)$ & 0.4991 & $0.5869(5)$ & $0.2930(9)$ & $1.0000(1)$ \\
4 & $0.3013(6)$ & $0.7593(2)$ & $0.3968(8)$ & $0.3209(7)$ & $0.9389(4)$ \\
5 & $0.3549(4)$ & $0.7289(4)$ & $0.4869(7)$ & $0.4304(4)$ & $0.8246(7)$ \\
6 & $0.5448(2)$ & $0.7359(3)$ & $0.7404(3)$ & $0.5448(3)$ & $1.0000(1)$ \\
7 & $0.1788(9)$ & $0.5516(8)$ & $0.3242(9)$ & 0.2881 & $0.6206(9)$ \\
8 & $0.2818(8)$ & $0.5325(9)$ & $0.5291(6)$ & $0.3052(8)$ & $0.9232(5)$ \\
9 & $0.3282(5)$ & $0.5526(7)$ & $0.5939(4)$ & $0.3845(5)$ & $0.8535(6)$ \\
10 & 0.1747 & $0.6498(5)$ & 0.2689 & $0.3722(6)$ & 0.4695 \\
& $(10)$ & & $(10)$ & $(10)$ \\
Mean & 0.3892 & 0.6564 & 0.5711 & 0.4492 & 0.8416 \\
SD & 0.2411 & 0.1537 & 0.2236 & 0.2163 & 0.1771 \\
Min & 0.1747 & 0.4991 & 0.2689 & 0.2881 & 0.4695 \\
Max & 1.0000 & 1.0000 & 1.0000 & 1.0000 & 1.0000 \\
\hline
\end{tabular}

efficiency scores, the additive efficiency score, and the multiplicative efficiency score, are given in the second to the fifth columns of Table 4, respectively. We then apply model (18) to determine the solution of the proposed model (13). The results of the efficiency scores are given in the sixth to the ninth columns of Table 4, respectively. All of the corresponding rank values for each DMU are listed in parentheses. The thirteenth to the sixteenth rows of Table 4 detail the descriptive statistics for the efficiency scores of 10 bank branches determined by models (9) and (18).

Comparing the proposed model (7) with Kao and Hwang's models (5) and (6), we have the following observations (see Tables 3 and 4): (i) among the nine DMUs with flexible efficiency decomposition, the results of the optimal efficiency decomposition as determined by the proposed model (7) are the same as the results from Kao and Hwang's model (5) for $\mathrm{DMU}_{10}$ and the results from Kao and Hwang's model (6) for the remaining eight DMUs. (ii) For each stage, the mean of individual efficiency scores determined by the proposed model (7) is between the means determined by Kao and Hwang's model (5) and model (6). This is because the individual efficiency scores determined by Kao and
Hwang's model (5) and model (6) are the extreme cases. (iii) For the individual efficiency score of each stage, the proposed model (7) presents more power of discrimination because of the higher standard deviation comparing with Kao and Hwang's models (5) and (6).

Comparing the proposed model (13) with Kao and Hwang's models (5) and (6), we have the following observations (see Tables 3 and 4): (i) For most DMUs, the ranks of the individual efficiency scores determined by the proposed model (16) lie between the ranks determined by Kao and Hwang's models (5) and (6), especially for $\mathrm{DMU}_{1}, \mathrm{DMU}_{5}$, $\mathrm{DMU}_{7}$, and $\mathrm{DMU}_{10}$, and the ranks of the second-stage efficiency scores determined by the proposed model (13) are same as the ranks determined by Kao and Hwang's models (5) and (6). Only for $\mathrm{DMU}_{5}, \mathrm{DMU}_{6}$, the ranks of the firststage efficiency score determined by the proposed model (13) lie in the neighborhood of the ranks determined by Kao and Hwang's models (5) or (6). (ii) For each stage, the mean and the standard deviation of individual efficiency scores determined by the proposed model (13) are between the means and the standard deviations determined by Kao and Hwang's models (5) and (6), respectively.

In the following, to obtain some interesting and valuable findings, we further compare the results determined by the proposed models with the results of the existing models.

\subsection{Comparing the Results Determined by the Proposed Model} (7) with the Results of Chiou et al.'s Model. The common feature of the proposed model (7) and Chiou et al.'s model is that the objective is maximizing the sum of the two individual efficiencies. The main difference between these models is that this objective is the main goal in Chiou et al.'s model but is a secondary goal in the proposed model (7). Thus, we select Chiou et al.'s model as the comparison object of the proposed model (7) in this section. We apply Chiou et al.'s model to determine the optimal additive efficiency decomposition of 10 bank branches, and the results, including two individual efficiency scores and the additive efficiency score, are presented in the second to the fourth columns of Table 5, respectively. To compare with the proposed method, the corresponding multiplicative efficiency score is also given in the fifth column of Table 5. All of the corresponding rank values for each DMU are listed in 
TABLE 4: Efficiency scores and ranks of 10 bank branches by the proposed models (7) and (13).

\begin{tabular}{|c|c|c|c|c|c|c|c|c|}
\hline \multirow{2}{*}{ DMU } & \multicolumn{4}{|c|}{ Proposed model (7) } & \multicolumn{4}{|c|}{ Proposed model (13) } \\
\hline & $E_{k}^{1 *}$ & $E_{k}^{2 *}$ & $E_{k a}^{*}$ & $E_{k p}^{*}$ & $E_{k}^{1 *}$ & $E_{k}^{2 *}$ & $E_{k a}^{*}$ & $E_{k p}^{*}$ \\
\hline 1 & $1.0000(1)$ & $1.0000(1)$ & $2.0000(1)$ & $1.0000(1)$ & $1.0000(1)$ & $1.0000(1)$ & $2.0000(1)$ & $1.0000(1)$ \\
\hline 2 & $0.5526(3)$ & $0.7861(8)$ & $1.3387(3)$ & $0.4344(3)$ & $0.5534(4)$ & $0.7850(3)$ & $1.3384(3)$ & $0.4344(3)$ \\
\hline 3 & $0.2930(9)$ & $1.0000(1)$ & $1.2930(4)$ & $0.2930(7)$ & $0.3961(10)$ & $0.7398(4)$ & $1.1359(6)$ & $0.2930(7)$ \\
\hline 4 & $0.3209(7)$ & $0.9389(4)$ & $1.2598(5)$ & $0.3013(6)$ & $0.5401(5)$ & $0.5579(8)$ & $1.0980(7)$ & $0.3013(6)$ \\
\hline 5 & $0.4304(5)$ & $0.8246(7)$ & $1.2550(6)$ & $0.3549(4)$ & $0.5797(3)$ & $0.6123(7)$ & $1.1919(4)$ & $0.3549(4)$ \\
\hline 6 & $0.5448(4)$ & $1.0000(1)$ & $1.5448(2)$ & $0.5448(2)$ & $0.6404(2)$ & $0.8508(2)$ & $1.4911(2)$ & $0.5448(2)$ \\
\hline 7 & $0.2881(10)$ & $0.6206(9)$ & $0.9087(10)$ & $0.1788(9)$ & $0.4199(8)$ & $0.4259(9)$ & $0.8457(10)$ & $0.1788(9)$ \\
\hline 8 & $0.3052(8)$ & $0.9232(5)$ & $1.2284(8)$ & $0.2818(8)$ & $0.4189(9)$ & $0.6728(6)$ & $1.0916(8)$ & $0.2818(8)$ \\
\hline 9 & $0.3845(6)$ & $0.8535(6)$ & $1.2380(7)$ & $0.3282(5)$ & $0.4686(7)$ & 0.7005 & $1.1690(5)$ & $0.3282(5)$ \\
\hline 10 & $0.6498(2)$ & $0.2689(10)$ & $0.9187(9)$ & $0.1747(10)$ & 0.5110 & $0.3419(10)$ & 0.8529 & $0.1747(10)$ \\
\hline Mean & 0.4769 & 0.8216 & 1.2985 & 0.3892 & 0.5528 & 0.6687 & 1.2215 & 0.3892 \\
\hline $\mathrm{SD}$ & 0.2231 & 0.2280 & 0.3096 & 0.2411 & 0.1756 & 0.1954 & 0.3354 & 0.2411 \\
\hline Min & 0.2881 & 0.2689 & 0.9087 & 0.1747 & 0.3961 & 0.3419 & 0.8457 & 0.1747 \\
\hline Max & 1.0000 & 1.0000 & 2.0000 & 1.0000 & 1.0000 & 1.0000 & 2.0000 & 1.0000 \\
\hline
\end{tabular}

TABle 5: Efficiency scores and ranks of 10 bank branches determined by the existing models.

\begin{tabular}{|c|c|c|c|c|c|c|c|c|}
\hline \multirow{2}{*}{ DMU } & \multicolumn{4}{|c|}{ Chiou et al.'s model } & \multicolumn{4}{|c|}{ Zhou et al.'s model } \\
\hline & $E_{k}^{1 *}$ & $E_{k}^{2 *}$ & $E_{k a}^{*}$ & $E_{k p}^{*}$ & $E_{k}^{1 *}$ & $E_{k}^{2 *}$ & $E_{k a}^{*}$ & $E_{k p}^{*}$ \\
\hline 1 & $1.0000(1)$ & $1.0000(1)$ & $2.0000(1)$ & $1.0000(1)$ & $1.0000(1)$ & $1.0000(1)$ & $2.0000(1)$ & $1.0000(1)$ \\
\hline 2 & $0.5526(3)$ & $0.7861(8)$ & $1.3387(3)$ & $0.4344(3)$ & $0.5534(4)$ & $0.7850(3)$ & $1.3384(3)$ & $0.4344(3)$ \\
\hline 3 & $0.2930(9)$ & $1.0000(1)$ & $1.2930(5)$ & $0.2930(7)$ & $0.3824(10)$ & $0.7661(4)$ & $1.1485(6)$ & $0.2930(7)$ \\
\hline 4 & $0.3070(6)$ & $0.9699(5)$ & $1.2769(6)$ & $0.2977(5)$ & $0.4936(5)$ & $0.6104(8)$ & $1.1040(7)$ & $0.3013(6)$ \\
\hline 5 & $0.4268(5)$ & $0.8293(7)$ & $1.2561(7)$ & $0.3539(4)$ & $0.5601(3)$ & $0.6337(7)$ & $1.1938(4)$ & $0.3549(4)$ \\
\hline 6 & $0.5448(4)$ & $1.0000(1)$ & $1.5448(2)$ & $0.5448(2)$ & $0.6332(2)$ & $0.8605(2)$ & $1.4937(2)$ & $0.5448(2)$ \\
\hline 7 & $0.2801(10)$ & $0.6316(9)$ & $0.9117(10)$ & $0.1769(9)$ & $0.3987(9)$ & $0.4485(9)$ & $0.8472(9)$ & $0.1788(9)$ \\
\hline 8 & $0.3052(7)$ & $0.9232(6)$ & $1.2284(8)$ & $0.2818(8)$ & $0.4032(8)$ & $0.6989(6)$ & $1.1021(8)$ & $0.2818(8)$ \\
\hline 9 & $0.2968(8)$ & $0.9988(4)$ & $1.2957(4)$ & $0.2965(6)$ & $0.4610(7)$ & $0.7119(5)$ & $1.1729(5)$ & $0.3282(5)$ \\
\hline 10 & $0.6498(2)$ & $0.2689(10)$ & $0.9187(9)$ & $0.1747(10)$ & $0.4918(6)$ & $0.3553(10)$ & $0.8471(10)$ & $0.1747(10)$ \\
\hline Mean & 0.4656 & 0.8408 & 1.3064 & 0.3854 & 0.5377 & 0.6870 & 1.2247 & 0.3892 \\
\hline SD & 0.2306 & 0.2353 & 0.3082 & 0.2425 & 0.1811 & 0.1886 & 0.3351 & 0.2411 \\
\hline Min & 0.2801 & 0.2689 & 0.9117 & 0.1747 & 0.3824 & 0.3553 & 0.8471 & 0.1747 \\
\hline Max & 1.0000 & 1.0000 & 2.0000 & 1.0000 & 1.0000 & 1.0000 & 2.0000 & 1.0000 \\
\hline
\end{tabular}

parentheses. The thirteenth to the sixteenth rows of Table 5 detail the descriptive statistics for the efficiency scores of 10 bank branches determined by the existing models.

Comparing the proposed model (7) with Chiou et al.'s model, we have the following observations (see Tables 4 and 5): (i) For $\mathrm{DMU}_{1}, \mathrm{DMU}_{2}, \mathrm{DMU}_{3}, \mathrm{DMU}_{6}, \mathrm{DMU}_{8}$, and $\mathrm{DMU}_{10}$, the results of the optimal efficiency decomposition as determined by the proposed model (7) are the same as the results from Chiou et al.'s model. For the remaining four DMUs, the optimal efficiency scores of the first/second stage as determined by the proposed model (7) are all greater/less than the scores of Chiou et al.'s model. (ii) The individual efficiencies $E_{k}^{1 *}$ and $E_{k}^{2 *}$, the additive efficiency $E_{k a}^{*}$, and the multiplicative efficiency $E_{k p}^{*}$ ranks of all of the DMUs determined by the proposed model (7) are not exactly the same as the ranks of Chiou et al.'s model. For example, the first/ second stage efficiency rank of $\mathrm{DMU}_{4}$ is $7 / 4$ in the proposed model (7), but its rank is $6 / 5$ in Chiou et al.'s model. The additive/multiplicative efficiency rank of $\mathrm{DMU}_{4}$ is $5 / 6$ in the proposed model (7), but its rank is $6 / 5$ in Chiou et al.'s model. (iii) For each stage, the means of individual efficiency scores determined by the proposed model (7) and Chiou et al.'s model are unequal. The mean of additive/multiplicative efficiency scores determined by the proposed model (7) is less/greater than the mean for Chiou et al.'s model, the results are consistent with the goal of their models. (iv) According to the standard deviation of the efficiency scores, the proposed model (7) presents more power of discrimination in the rank of the additive efficiency scores but less power of discrimination in the rank of the two individual efficiency scores and the multiplicative efficiency scores.

To further compare the results determined by the proposed model (7) with the results of Chiou et al.'s model, Spearman's rank correlation coefficient test is used to assess the rank correlations of the efficiency scores for two models. The results are illustrated as follows: (i) Spearman's rank correlation coefficients of $E_{k}^{1 *}, E_{k}^{2 *}, E_{k a}^{*}$, and $E_{k p}^{*}$ for the proposed model (7) and Chiou et al.'s model are calculated as $0.964,0.964,0.927$, and 0.988 , respectively. For the $\alpha=$ 0.05 significant level, all correlation coefficients are statistically significant because the threshold value is 0.564 . This result shows that the rank that is determined by the proposed 
model (7) has the same direction as that of Chiou et al.'s model. (ii) Spearman's rank correlation coefficients for $E_{k a}^{*}$ and $E_{k p}^{*}$ that are obtained by the proposed model (7) are calculated as 0.879 , which is equal to that of Chiou et al.'s model. For the $\alpha=0.05$ significant level, the correlation coefficients are statistically significant because the threshold value is 0.564 .

Spearman's rank correlation coefficients tests further show that the multiplicative and additive models are two different models but that they are equally valid ways of aggregating the components of a two-stage process, as Chen et al. explained [21]. The advantages of the proposed model (7) over Chiou et al.'s model are summarized as follows: (i) Chiou et al.'s model is not a convex optimization for the objective function is not concave, and local optima may exist, which are not globally optimal [24]. The proposed model (7) can be converted into a linear programming problem for which the optimal solution is global in contrast to the nonlinear solution. (ii) The proposed model (7) can be easily extended to the case for which the relative importance of the performances of the two stages to the overall performance of the DMU is considered in the secondary goal without increasing the difficulty for solving it.

The comparisons above offer some valuable insights for the choice of the models: (i) If the two-stage DEA model is used to determine the efficiencies scores of all of the DMUs, then the choice of the models depends on the decision maker's preference in the particular application. (ii) If the two-stage DEA model is used to determine the efficiencies ranks of all of the DMUs, then the multiplicative model should be selected. The rank that is determined by the multiplicative model and additive model has the same direction, and the multiplicative model is easy to solve to optimality.

4.2. Comparing the Results Determined by the Proposed Model (13) with the Results of Zhou et al.'s Model. The main purpose of the proposed model (13) and Zhou et al.'s model is determining the efficiency decomposition for the two stages while keeping the multiplicative efficiency unchanged. In the following, we will compare the results of the efficiency decomposition determined by the proposed model (13) with the results of Zhou et al.'s model. We apply Zhou et al.'s model to determine the two individual efficiency scores of 10 bank branches, and the results are presented in the sixth and the seventh columns of Table 4 , respectively. To compare with the proposed method, the corresponding additive efficiency score and multiplicative efficiency score are also given in the eighth and the ninth columns of Table 4, respectively.

Comparing the proposed model (13) with Zhou et al.'s model, we have the following observations (see Tables 3 and 4): (i) according to the results of Zhou et al.'s model and the proposed model (13), only $\mathrm{DMU}_{1}$ is relatively efficient and the remaining nine DMUs are relatively inefficient in both stages and in the whole system. Particularly, the efficiency scores for the second stage of $\mathrm{DMU}_{3}$ and $\mathrm{DMU}_{6}$ are less than 1 , which is different from Chiou et al.'s model and the proposed model (7). Thus, the decomposition of the multiplicative efficiency using the proposed model (13) and Zhou et al.'s model can help identify the stage that causes inefficiency. (ii) The second-stage efficiency ranks that are determined by the proposed model (13) for all DMUs are exactly the same as the ranks for Zhou et al.'s model. For the first-stage efficiency and the additive efficiency, only two DMU ranks are different in the two models. For example, the first-stage efficiency rank of $\mathrm{DMU}_{7}$ is eight in the proposed model (13), but its rank is nine in Zhou et al.'s model. The additive efficiency rank of $\mathrm{DMU}_{10}$ is nine in the proposed model (13), but its rank is ten in Zhou et al.'s model. (iii) For each stage, the mean of the individual efficiency scores determined by the proposed model (13) and Zhou et al.'s model are not equal. According to the standard deviation of the efficiency scores, the proposed model (13) presents more power of discrimination in the rank of the second-stage efficiency score, but less power of discrimination in the rank of the first-stage efficiency score.

Spearman's rank correlation coefficient test is also used to assess the rank correlations of the efficiency scores for two models, and the result shows that the rank that is determined by the proposed model (13) has the same direction as that of Zhou et al.'s model. The main advantage of the proposed model (13) over Zhou et al.'s model is that the proposed model (13) can be easily extended to the weighted model in which the relative importance of the two stages to the overall system is considered in the secondary goal.

\section{Conclusions}

Kao and Hwang proposed a multiplicative two-stage DEA model by taking into account the series relationship of the two subprocesses within the whole production process, and the overall efficiency is the product of the efficiencies of the two subprocesses. However, as Kao and Hwang mentioned, the optimal weights that are solved from their model may not be unique. Consequently, some flexibility exists in the efficiency decomposition between the two stages [4].

To solve this problem, we maximize the sum of the two individual efficiencies and simultaneously maximize the two individual efficiencies, respectively, as a secondary goal in the multiplicative two-stage DEA model to select the decomposition of the overall efficiency from the flexible decompositions. The proposed models with their different secondary goals are applied to a numerical example for the efficiency decomposition of 10 bank branches, and the results are compared with the results of the existing models.

The contributions of the paper are summarized as follows: (i) the proposed methods provide more alternative ways for the decomposition of the overall efficiency in the multiplicative two-stage DEA model. (ii) The proposed models are easy to solve to optimality and can be easily extended to the case for which the weight of the two stages is considered in the secondary goal without increasing the difficulty for solving it. (iii) Some interesting and valuable findings for the choice of the models are obtained by the comparisons of the results. 


\section{Data Availability}

The data used to support the findings of this study are included within the article.

\section{Conflicts of Interest}

The authors declare that there are no conflicts of interest regarding the publication of this article.

\section{References}

[1] A. Charnes, W. W. Cooper, and E. Rhodes, "Measuring the efficiency of decision making units," European Journal of Operational Research, vol. 2, no. 6, pp. 429-444, 1978.

[2] W. D. Cook, L. Liang, and J. Zhu, "Measuring performance of two-stage network structures by DEA: a review and future perspective," Omega, vol. 38, no. 6, pp. 423-430, 2010.

[3] L. M. Seiford and J. Zhu, "Profitability and marketability of the top 55 U.S. Commercial banks," Management Science, vol. 45, no. 9, pp. 1270-1288, 1999.

[4] C. Kao and S.-N. Hwang, "Efficiency decomposition in twostage data envelopment analysis: an application to non-life insurance companies in taiwan," European Journal of Operational Research, vol. 185, no. 1, pp. 418-429, 2008.

[5] Y.-C. Chiou, L. W. Lan, and B. T. H. Yen, "A joint measurement of efficiency and effectiveness for non-storable commodities: integrated data envelopment analysis approaches," European Journal of Operational Research, vol. 201, no. 2, pp. 477-489, 2010.

[6] C. Chen, J. Zhu, J.-Y. Yu, and H. Noori, “A new methodology for evaluating sustainable product design performance with two-stage network data envelopment analysis," European Journal of Operational Research, vol. 221, no. 2, pp. 348-359, 2012.

[7] C.-H. Yang, H.-Y. Lin, and C.-P. Chen, "Measuring the efficiency of NBA teams: additive efficiency decomposition in two-stage DEA," Annals of Operations Research, vol. 217, no. 1, pp. 565-589, 2014.

[8] C.-w. Huang, F. N. Ho, and Y.-h. Chiu, "Measurement of tourist hotels' productive efficiency, occupancy, and catering service effectiveness using a modified two-stage DEA model in taiwan," Omega, vol. 48, pp. 49-59, 2014.

[9] P. Yin, J. Chu, J. Wu, J. Ding, M. Yang, and Y. Wang, "A DEAbased two-stage network approach for hotel performance analysis: an internal cooperation perspective," Omega-international Journal of Management Science, vol. 93, Article ID 102035, 2020.

[10] K. Wang, W. Huang, J. Wu, and Y.-N. Liu, "Efficiency measures of the Chinese commercial banking system using an additive two-stage DEA," Omega, vol. 44, pp. 5-20, 2014.

[11] P. Wanke and C. Barros, "Two-stage DEA: an application to major Brazilian banks," Expert Systems with Applications, vol. 41, no. 5, pp. 2337-2344, 2014.

[12] I. C. Henriques, V. A. Sobreiro, H. Kimura, and E. B. Mariano, "Two-stage DEA in banks: terminological controversies and future directions," Expert Systems with Applications, vol. 161, Article ID 113632, 2020.

[13] E. Wang, Z. Shen, N. Alp, and N. Barry, "Benchmarking energy performance of residential buildings using two-stage multifactor data envelopment analysis with degree-day based simple-normalization approach," Energy Conversion and Management, vol. 106, pp. 530-542, 2015.
[14] Q. Zhu, X. Li, F. Li, J. Wu, and D. Zhou, "Energy and environmental efficiency of China's transportation sectors under the constraints of energy consumption and environmental pollutions," Energy Economics, vol. 89, Article ID 104817, 2020.

[15] A. Tajbakhsh and E. Hassini, "A data envelopment analysis approach to evaluate sustainability in supply chain networks," Journal of Cleaner Production, vol. 105, pp. 74-85, 2015.

[16] M. Tavana, M. A. Kaviani, D. Di Caprio, and B. Rahpeyma, “A two-stage data envelopment analysis model for measuring performance in three-level supply chains," Measurement, vol. 78, pp. 322-333, 2016.

[17] M.-M. Yu, L.-H. Chen, and B. Hsiao, "A fixed cost allocation based on the two-stage network data envelopment approach," Journal of Business Research, vol. 69, no. 5, pp. 1817-1822, 2016.

[18] Q. Zhu, X. Li, F. Li, J. Wu, and J. Sun, "Analyzing the sustainability of China's industrial sectors: a data-driven approach with total energy consumption constraint," Ecological Indicators, vol. 122, Article ID 107235, 2021.

[19] C. Kao, "Network data envelopment analysis: a review," European Journal of Operational Research, vol. 239, no. 1, pp. 1-16, 2014.

[20] G. E. Halkos, N. G. Tzeremes, and S. A. Kourtzidis, "A unified classification of two-stage DEA models," Surveys in Operations Research and Management Science, vol. 19, no. 1, pp. 1-16, 2014.

[21] Y. Chen, W. D. Cook, N. Li, and J. Zhu, "Additive efficiency decomposition in two-stage DEA," European Journal of Operational Research, vol. 196, no. 3, pp. 1170-1176, 2009.

[22] Y.-M. Wang and K.-S. Chin, "Some alternative DEA models for two-stage process," Expert Systems with Applications, vol. 37, no. 12, pp. 8799-8808, 2010.

[23] C. Guo, R. Abbasi Shureshjani, A. A. Foroughi, and J. Zhu, "Decomposition weights and overall efficiency in two-stage additive network DEA," European Journal of Operational Research, vol. 257, no. 3, pp. 896-906, 2017.

[24] S. Lim and J. Zhu, "Integrated data envelopment analysis: global vs. Local optimum," European Journal of Operational Research, vol. 229, no. 1, pp. 276-278, 2013.

[25] L. Liang, F. Yang, W. D. Cook, and J. Zhu, "DEA models for supply chain efficiency evaluation," Annals of Operations Research, vol. 145, no. 1, pp. 35-49, 2006.

[26] G. E. Halkos, N. G. Tzeremes, and S. A. Kourtzidis, "Weight Assurance region in two-stage additive efficiency decomposition DEA model: an application to school data," Journal of the Operational Research Society, vol. 66, no. 4, pp. 696-704, 2015.

[27] C. Kao and S.-N. Hwang, "Decomposition of technical and scale efficiencies in two-stage production systems," European Journal of Operational Research, vol. 211, no. 3, pp. 515-519, 2011.

[28] Y. Chen and J. Zhu, "Measuring information technology's indirect impact on firm performance," Information Technology and Management, vol. 5, no. 1, pp. 9-22, 2004.

[29] L. Liang, W. D. Cook, and J. Zhu, "DEA models for two-stage processes: game approach and efficiency decomposition," Naval Research Logistics, vol. 55, no. 7, pp. 643-653, 2008.

[30] S.-T. Liu, "A note on efficiency decomposition in two-stage data envelopment analysis," European Journal of Operational Research, vol. 212, no. 3, pp. 606-608, 2011.

[31] Z. Zhou, L. Sun, W. Yang, W. Liu, and C. Ma, "A bargaining game model for efficiency decomposition in the centralized 
model of two-stage systems," Computers \& Industrial Engineering, vol. 64, no. 1, pp. 103-108, 2013.

[32] S. Dimitrov and W. Sutton, "Promoting symmetric weight selection in data envelopment analysis: a penalty function approach," European Journal of Operational Research, vol. 200, no. 1, pp. 281-288, 2010.

[33] G. R. Jahanshahloo, F. Hosseinzadeh Lotfi, Y. Jafari, and R. Maddahi, "Selecting symmetric weights as a secondary goal in DEA cross-efficiency evaluation," Applied Mathematical Modelling, vol. 35, no. 1, pp. 544-549, 2011.

[34] I. Contreras, "Optimizing the rank position of the DMU as secondary goal in DEA cross-evaluation," Applied Mathematical Modelling, vol. 36, no. 6, pp. 2642-2648, 2012.

[35] M. Soltanifar and S. Shahghobadi, "Selecting a benevolent secondary goal model in data envelopment analysis crossefficiency evaluation by a voting model," Socio-Economic Planning Sciences, vol. 47, no. 1, pp. 65-74, 2013.

[36] M. Tavana, K. Khalili-Damghani, F. J. Santos Arteaga, R. Mahmoudi, A. Hafezalkotob, and A. Hafezalkotob, "Efficiency decomposition and measurement in two-stage fuzzy DEA models using a bargaining game approach," Computers \& Industrial Engineering, vol. 118, pp. 394-408, 2018.

[37] Q. An, F. Meng, S. Ang, and X. Chen, "A new approach for fair efficiency decomposition in two-stage structure system," Operational Research, vol. 18, no. 1, pp. 257-272, 2018.

[38] L. Fang, "Stage efficiency evaluation in a two-stage network data envelopment analysis model with weight priority," Omega-international Journal of Management Science, vol. 97, Article ID 102081, 2020.

[39] L. Liang, J. Wu, W. D. Cook, and J. Zhu, "Alternative secondary goals in DEA cross-efficiency evaluation," International Journal of Production Economics, vol. 113, no. 2, pp. 1025-1030, 2008.

[40] Y.-M. Wang and K.-S. Chin, "Some alternative models for DEA cross-efficiency evaluation," International Journal of Production Economics, vol. 128, no. 1, pp. 332-338, 2010.

[41] J. Wu, J. Chu, J. Sun, Q. Zhu, and L. Liang, "Extended secondary goal models for weights selection in DEA cross-efficiency evaluation," Computers \& Industrial Engineering, vol. 93, pp. 143-151, 2016.

[42] M. Jiménez and A. Bilbao, "Pareto-optimal solutions in fuzzy multi-objective linear programming," Fuzzy Sets and Systems, vol. 160, no. 18, pp. 2714-2721, 2009.

[43] M. Jiménez, "Ranking fuzzy numbers through the comparison of its expected intervals," International Journal of Uncertainty, Fuzziness and Knowledge-Based Systems, vol. 4, no. 4, pp. 379-388, 1996.

[44] C.-C. Lin, "A weighted max-min model for fuzzy goal programming," Fuzzy Sets And Systems, vol. 142, no. 3, pp. 407-420, 2004.

[45] F. Yang, D. Wu, L. Liang, G. Bi, and D. D. Wu, "Supply chain DEA: production possibility set and performance evaluation model," Annals of Operations Research, vol. 185, no. 1, pp. 195-211, 2011. 